\title{
Differential Responsiveness in VEGF Receptor Subtypes to Hypoxic Stress in Various Tissues of Plateau Animals
}

\author{
Hui-Chun XIE ${ }^{1,2}$, Jin-Gang LI ${ }^{1}$, Jian-Ping $\mathrm{HE}^{1}$ \\ ${ }^{1}$ College of Life Science, Shaanxi Normal University, Xi'an, China, ${ }^{2}$ Key Laboratory of Education \\ Ministry on Environments and Resources in Tibetan Plateau, Qinghai Normal University, Xining, \\ China
}

Received June 13, 2016

Accepted July 22, 2016

On-line December 16, 2016

\section{Summary}

With hypoxic stress, hypoxia-inducible factor-1a (HIF-1a) and vascular endothelial growth factor (VEGF) are elevated and their responses are altered in skeletal muscles of plateau animals [China Qinghai-Tibetan plateau pikas (Ochotona curzoniae)] as compared with control animals [normal lowland Sprague-Dawley (SD) rats]. The results indicate that HIF-1a and VEGF are engaged in physiological functions under hypoxic environment. The purpose of the current study was to examine the protein levels of VEGF receptor subtypes (VEGFRs: VEGFR-1, VEGFR-2 and VEGFR-3) in the end organs, namely skeletal muscle, heart and lung in response to hypoxic stress. ELISA and Western blot analysis were employed to determine HIF-1a and the protein expression of VEGFRs in control animals and plateau pikas. We further blocked HIF-1a signal to determine if HIF-1a regulates alternations in VEGFRs in those tissues. We hypothesized that responsiveness of VEGFRs in the major end organs of plateau animals is differential with insult of hypoxic stress and is modulated by low oxygen sensitive HIF-1a. Our results show that hypoxic stress induced by exposure of lower $\mathrm{O}_{2}$ for $6 \mathrm{~h}$ significantly increased the levels of VEGFR-2 in skeletal muscle, heart and lung and the increases were amplified in plateau pikas. Our results also demonstrate that hypoxic stress enhanced VEGFR-3 in lungs of plateau animals. Nonetheless, no significant alternations in VEGFR-1 were observed in those tissues with hypoxic stress. Moreover, we observed decreases of VEGFR-2 in skeletal muscle, heart and lung; and decreases of VEGFR-3 in lung following HIF-1a inhibition. Overall, our findings suggest that in plateau animals 1 ) responsiveness of VEGFRs is different under hypoxic environment; 2) amplified VEGFR-2 response appears in skeletal muscle, heart and lung, and enhanced VEGFR-3 response is mainly observed in lung; 3) HIF-1a plays a regulatory role in the levels of VEGFRs. Our results provide the underlying cellular and molecular mechanisms responsible for hypoxic environment in plateau animals, having an impact on research of physiological and ecological adaptive responses to acute or chronic hypoxic stress in humans who living at high attitude and who live at a normal sea level but suffer from hypoxic disorders.

\section{Key words}

Hypoxic stress • HIF-1a • VEGFRs • Skeletal muscle • Heart • Lung

\section{Corresponding author}

J.-P. He, College of Life Science, Shaanxi Normal University, 620 West Chang'an Avenue, Xi'an 710119, China. E-mail: hejianping64@163.com

Plateau pikas (Ochotona curzoniae) are the predominant species of small native mammals to live on the China Qinghai-Tibetan plateau alpine meadow with an altitude of approximately $3000 \mathrm{~m}$. They are completely acclimatized to the hypoxic environment (Du and $\mathrm{Li} \mathrm{1982).} \mathrm{Also,} \mathrm{as} \mathrm{a} \mathrm{unique} \mathrm{model} \mathrm{of} \mathrm{hypoxic}$ tolerance plateau pikas have been used to study the physiological and ecological adaptive responses to acute or chronic hypoxic stress as well as the underlying cellular and molecular mechanisms related to hypoxic environment (Chen et al. 2007). 
Hypoxia inducible factor-1 (HIF-1) has an important contribution to physiological changes of homeostasis under conditions of oxygen deprivation (Ceradini et al. 2004, Manalo et al. 2005). Accumulated subunit HIF-1 $\alpha$ modulates the expression of several target genes in protecting tissues against hypoxia (Ceradini et al. 2004, Manalo et al. 2005). In addition, with inadequate oxygen supply vascular endothelial growth factor (VEGF) is considered as a signal protein produced by cells and further plays an important role in improving vascular functions and vasculogenesis and thereby leading to restoring the oxygen supply to tissues (Kim and Byzova 2014). Also, HIF-1 $\alpha$ is engaged in many cellular pathways to play a functional role, including glucose uptake and carbohydrate metabolism (Semenza 2001). In plateau animals, these metabolic mechanisms are adjusted to meet hypoxic environment (Chen et al. 2007). Our recent study suggests that responses of HIF-1 $\alpha$ and VEGF to hypoxic stress are blunted in skeletal muscle of plateau pikas (Xie et al. 2014).

VEGF regulates vascular development, angiogenesis and lymphangiogenesis by binding to a number of VEGFRs (Holmes et al. 2007, Stuttfeld and Ballmer-Hofer 2009). There are three main subtypes of VEGFR, numbered 1, 2 and 3 (VEGFR-1, VEGFR-2 and VEGFR-3). Therefore, in the current study, we examined the protein expression of these VEGFR subtypes in the end organs, namely skeletal muscle, heart and lung of lowland control animals and plateau pikas in response to hypoxic stress induced by exposure of lower $\mathrm{O}_{2}$. Moreover, we systemically injected an inhibitor of HIF-1 $\alpha$ [(2-methoxyestradiol (2-MeOE2)] and then examined the levels of VEGFRs in those tissues to determine the effects of HIF- $1 \alpha$ on VEGFRs. We hypothesized that responses of VEGFRs are amplified in the end organs of plateau pikas during hypoxia as compared with lowland control animals. We also hypothesized that HIF-1 $\alpha$ contributes to expression of VEGFRs with hypoxic stress.

All procedures outlined in this study were in accordance with the Guide for the Care and Use of Laboratory Animals (US NIH), and approved by the animal Care Committee of our institution. Forty-five healthy adult male plateau pikas (150-200 g, obtained from the Qinghai Alpine Meadow, 3000 m altitude) and sixty Sprague-Dawley (SD) rats (from Laboratory Animal Center of this Institute) were used in this study. Hypoxic stress was performed in a chamber of $70 \times 70 \times 50 \mathrm{~cm}$ ventilated with a mixed gas $\left(\mathrm{O}_{2} / \mathrm{N}_{2} \%\right)$ of
$21 \%(\sim$ sea level $)$ as control, and of $10.8 \% \mathrm{O}_{2}(\sim 5000 \mathrm{~m}$ altitude) as hypoxic stimulator (Chen et al. 2007), which was controlled by oxygen sensor (Innovative Instruments Co., USA). In additional experiments, 2-MeOE2 (15 mg, Selleck Chem. Co., USA) was intraperitoneally injected in animals with hypoxia. All the animals were kept in the chambers for $6 \mathrm{~h}$, and then decapitated following overdose of isoflurane in order to remove the glycolytic portion of the gastrocnemius muscle, heart and lungs.

The levels of HIF-1 $\alpha$ (Abcam Co., UK) were determined using a two-site immunoenzymatic assay (ELISA) according to the provided description and modification. Briefly, polystyrene 96-well microtitel immunoplates were coated with affinity-purified rabbit anti-HIF-1 $\alpha$ antibody. Parallel wells were coated with purified rabbit $\mathrm{IgG}$ for evaluation of nonspecific signal. After overnight incubation at room temperature and $2 \mathrm{~h}$ of incubation with the coating buffer containing $50 \mathrm{mM}$ carbonate buffer ( $\mathrm{pH} 9.5$ ) in $2 \% \mathrm{BSA}$, plate were washed with $50 \mathrm{mM}$ Tris-HCl. After extensive washing, the diluted samples and the HIF-1 $\alpha$ standard solutions were distributed in each plate and left at room temperature overnight. The plates were washed and incubated with anti-HIF-1 $\alpha$-galactosidase per well. Then, the plates were washed and incubated with substrate solution. After an incubation of $2 \mathrm{~h}$ at $37^{\circ} \mathrm{C}$, the optical density was measured at $575 \mathrm{~nm}$ of wavelength using an ELISA reader (Dynatech, USA).

The protein expression of VEGFRs was determined by using a standard Western blot analysis. In brief, the tissues from individual rats were sampled. Total protein was then extracted by homogenizing sample in ice-cold immunoprecipitation assay buffer with protease inhibitor cocktail kit. The lysates were centrifuged and the supernatants were collected for measurements of protein concentrations using a bicinchoninic acid assay reagent kit. After being denatured by heating at $95{ }^{\circ} \mathrm{C}$ in an SDS sample buffer, the supernatant samples were loaded onto 4-20\% Mini-Protean TGX Precast gels and then electrically transferred to a polyvinylidene fluoride membrane. Membranes were incubated with the rabbit anti-VEGFR-1, anti-VEGFR-2 and anti-VEGFR-3 primary antibodies (1:500, obtained from Neuromics and Abcam Co., USA). After being fully washed, the membrane was incubated with horseradish peroxidaselinked anti-rabbit secondary antibody (1:250) and visualized for immunoreactivity. The membrane was also processed to detect $\beta$-actin for equal loading. The bands recognized by the primary antibody were visualized by 
exposure of the membrane onto an X-ray film. The film was then scanned and the optical densities of protein bands were analyzed using the Scion image software.

All data were analyzed using a two-way repeated-measure analysis of variance due to two interventions (normoxic and hypoxic conditions) in two groups of animals. Values are presented as means \pm standard error (SE). For all analyses, differences were considered significant at $P<0.05$. All statistical analyses were performed by using SPSS for Windows version 15.0 (SPSS Inc., Chicago, USA).

In this study, we first examined the levels of HIF-1 $\alpha$ in skeletal muscle, heart and lung tissues of SD control rats and pikas. Figure 1 shows that under normoxic condition HIF-1 $\alpha$ levels in these tissues of pikas were lower than those in SD rats, but there were no significant differences observed in HIF-1 $\alpha$ between the two groups. Moreover, Figure 1 shows that hypoxic stress significantly increased the levels of HIF-1 $\alpha$ in the glycolytic skeletal muscle, heart and lung tissues of SD rats and pikas as compared with normoxic conditions. However, the increases of HIF-1 $\alpha$ were blunted in pikas in these tissues. We have also examined the levels of HIF-1 $\alpha$ after 2-MeOE2 in order to confirm its effectiveness in this experiment. In skeletal muscle, heart and lung of SD control rats and pikas HIF-1 $\alpha$ response to hypoxia was attenuated by $2-\mathrm{MeOE} 2$.
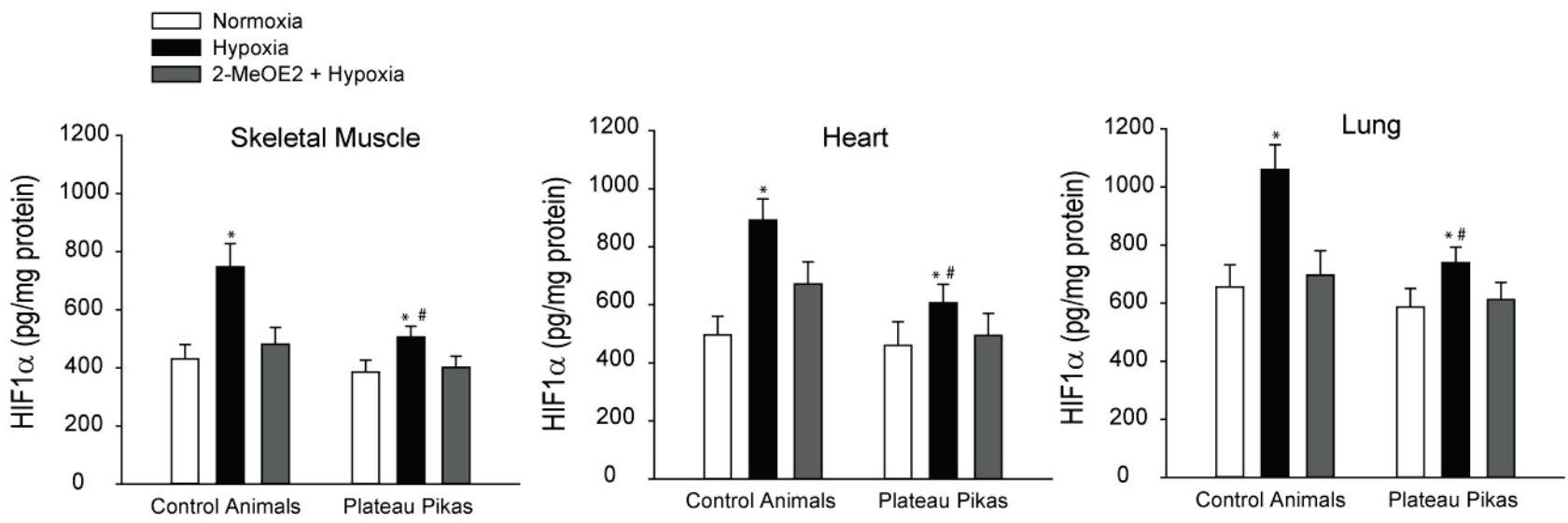

Fig. 1. Hypoxic stress increased the levels of HIF-1a in the glycolytic skeletal muscle, heart and lung of control animals ( $n=20$ for each condition) and plateau pikas ( $n=15$ for each condition). The increases of HIF-1a were smaller in pikas than that in control animals. Inhibiting HIF-1a by injecting 2-MeOE2 also attenuated expression of HIF-1a. $* P<0.05$ vs. normoxic and hypoxic conditions with 2-MeOE2. \# $P<0.05$ vs. control animals under hypoxia.

Figure 2 demonstrates that hypoxic stress increased expression of VEGFR-2 and VEGFR-3, but not VEGFR-1 in the glycolytic skeletal muscle, heart and lung of SD rats and pikas as compared with control conditions. In these tissues, increases of VEGFR-2 were amplified in pikas. In lung, an increase of VEGFR-3 was greater in pikas. However, in skeletal muscle and heart, there were no significant differences in increased amplitude of VEGFR-3 between two groups of animals. Figure 2 further demonstrates that inhibiting HIF-1 $\alpha$ by injecting 2-MeOE2 significantly attenuated expression of VEGFs induced by hypoxic stimulation compared with hypoxic conditions without 2-MeOE2.

Skeletal muscles are made of different fibers, namely oxidative and glycolytic muscle fibers, according to the relative involvement of energy metabolisms
(Lieber 2002). HIF-1 $\alpha$ has been considered as a critical sensor of cellular oxygen engaged in energy metabolisms (Ceradini et al. 2004, Manalo et al. 2005). In a prior study, the protein expression of the regulatory HIF- $1 \alpha$ was examined in oxidative and glycolytic muscles of the hindlimb. A higher level of HIF-1 $\alpha$ expression was observed in predominantly glycolytic muscles than in predominantly oxidative muscle under normoxic conditions (Pisani and Dechesne 2005). The changes in HIF-1 $\alpha$ content according to skeletal muscle fiber types are related to the myoglobin content (Grange et al. 2001). Oxidative fiber muscles with a high level of myoglobin have a low HIF-1 $\alpha$ content (Lin et al. 2002). Likewise, glycolytic fibers expressing a low level of myoglobin (Vogt et al. 2001) have a high HIF-1 $\alpha$ content. 


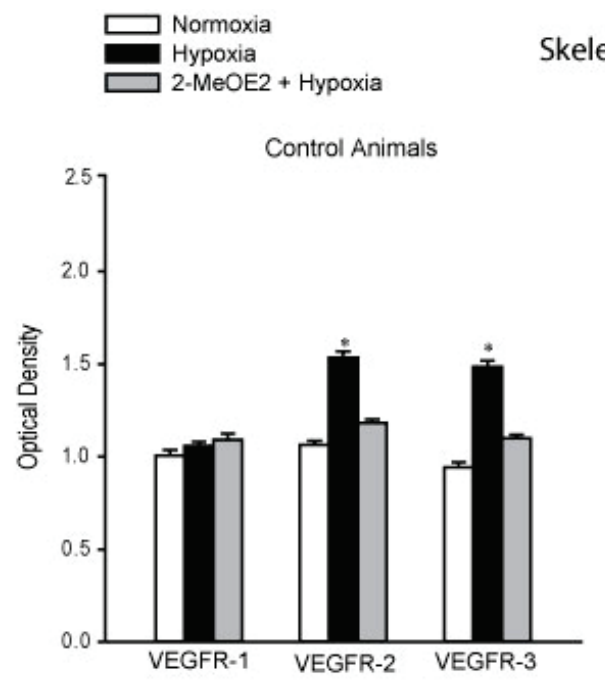

Skeletal Muscle
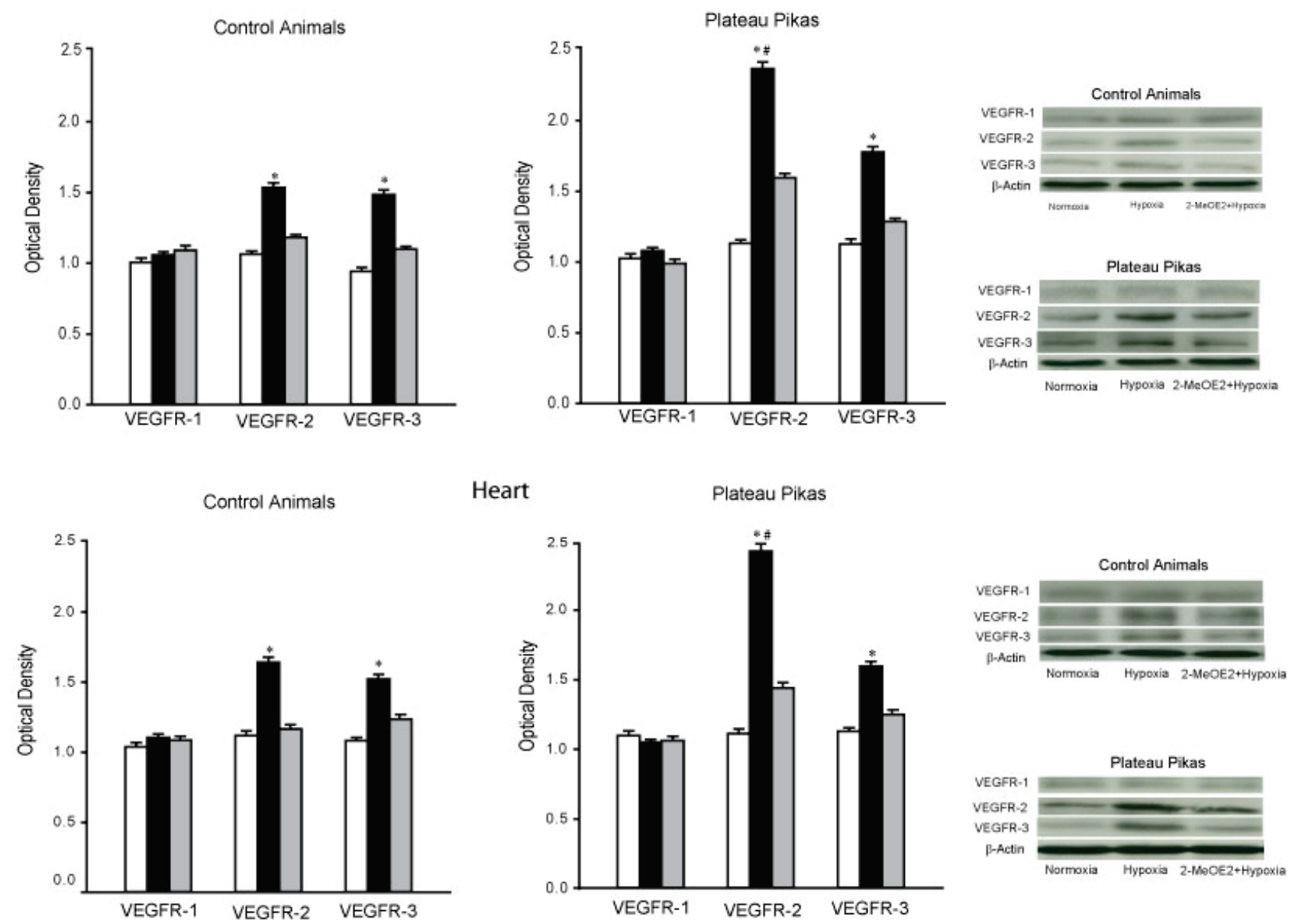

Heart

\section{Plateau Pikas}
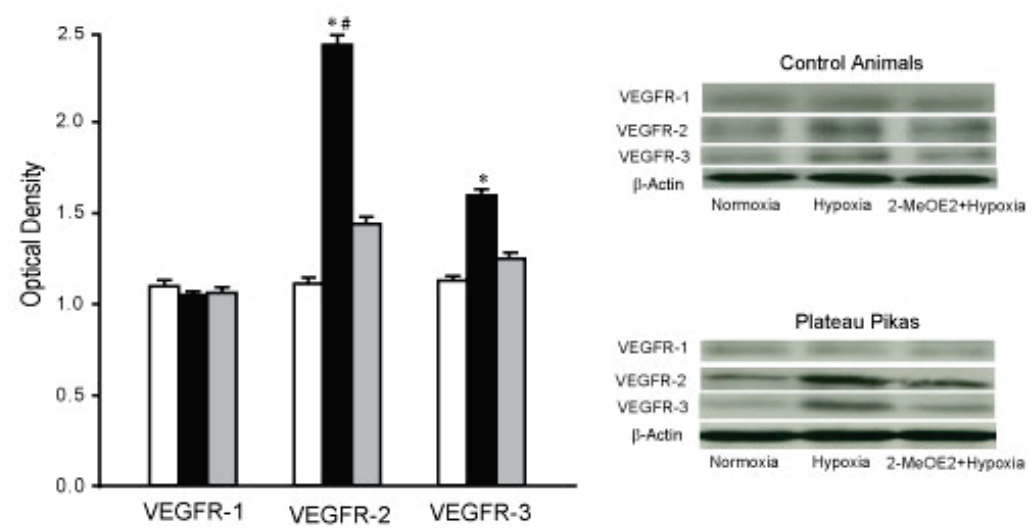

Lung
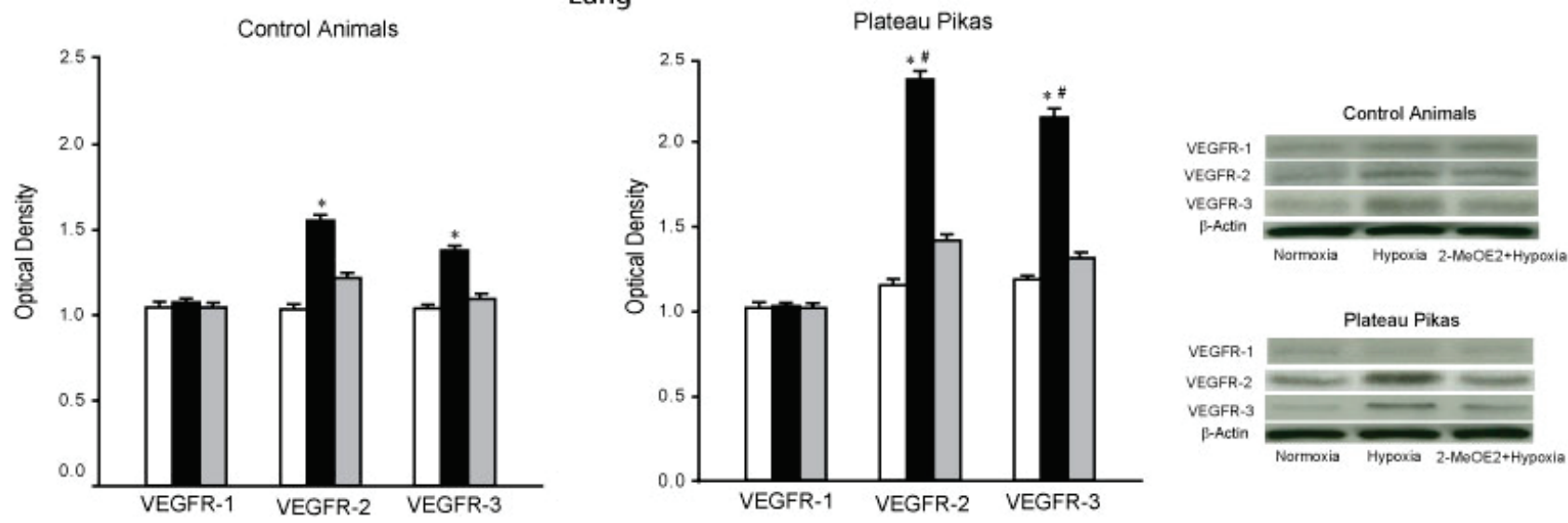

Fig. 2. Hypoxic stress increased the levels of VEGFR-2 and VEGFR-3 in the glycolytic skeletal muscle, heart and lung of control animals ( $n=20$ for each condition) and plateau pikas ( $n=15$ for each condition). Left and middle panels indicate averaged data; and right panels represent typical bands. The increases of VEGFR-2 were amplified in these tissues of plateau pikas. Increased VEGFR-3 was observed in lung of plateau pikas. There were no significant alternations in VEGFR-1 of those tissues under hypoxic stress. Inhibiting HIF-1a formation attenuated enhancement of VEGFR-2 and VEGFR-3 in skeletal muscle, heart and lung. $* P<0.05$ vs. normoxic and hypoxic conditions with 2-MeOE2. \# $P<0.05$ vs. control animals under hypoxia.

Thus, in the current study we first determined the protein levels of HIF-1 $\alpha$ in glycolytic muscles. Consistent with our previous findings (Xie et al. 2014), our data show that the protein levels of HIF-1 $\alpha$ in response to hypoxic stress were impaired in glycolytic muscles of plateau pikas compared with lowland animals. 
Our prior study has also shown that under hypoxic stress VEGF response is lower and HIF-1 $\alpha$ plays a role in regulating expression of skeletal muscle VEGF since a close relation is observed between HIF- $1 \alpha$ and VEGF in the skeletal muscles with hypoxic stress (Xie et al. 2014). Using the same intervention we further examined the expression of VEGFRs in glycolytic portion of skeletal muscles. We also determined the VEGFRs in the heart and lungs.

The function of VEGFR-1 is less defined, although it is required for the recruitment of hematopoietic stem cells and the migration of monocytes and macrophages (Holmes et al. 2007, Stuttfeld and Ballmer-Hofer 2009). VEGFR-2 appears to mediate almost all of the known cellular responses to VEGF and it is critical for vascular endothelial cell development and regulates vascular endothelial function (Holmes et al. 2007). VEGFR-3 regulates lymphatic endothelial cell function and mediates lymphangiogenesis in response to VEGF (Holmes et al. 2007, Stuttfeld and Ballmer-Hofer 2009). Considerable evidence shows VEGFR-2 specific intracellular signal cascades leading to proliferation, migration, survival and increased permeability, each of which contributes to the angiogenic response (Holmes et al. 2007).

Results of our current study show that hypoxic stress significantly increased the levels of VEGFR-2 in skeletal muscle, heart and lung and the increases were amplified in plateau pikas. Our results also demonstrate that hypoxic stress enhanced VEGFR-3 in lungs of plateau animals. Nonetheless, no significant alternations in VEGFR-1 of those tissues were observed with hypoxic stress. These results indicate that VEGFRs are selectively upregulated in different tissues in response to a lower level of VEGF under hypoxic stress. Moreover, we observed that blocking HIF-1 $\alpha$ formation decreased enhancement of VEGFR-2 in skeletal muscle, heart and lung and attenuated increases of VEGFR-3 in lung. This suggests that HIF- $1 \alpha$ is necessary in involvement of VEGFRs responsiveness. It should be noted that besides HIF- $1 \alpha$ and VEGFRs, other transcription factors such as $\mathrm{NF}-\mathrm{KB}$ and p-STAT3 etc. are also engaged in adaptive responses to hypoxic condition (Gu et al. 2011).

Overall, our findings suggest that in plateau animals 1) responsiveness of VEGFRs is different under hypoxic environment; 2) amplified VEGFR-2 response appears in skeletal muscle, heart and lung and enhanced VEGFR-3 response is mainly observed in lung; 3) HIF-1 $\alpha$ plays a regulatory role in the levels of VEGFRs. Our results provide the underlying cellular and molecular mechanisms responsible for hypoxic environment in plateau animals, having an impact on research of physiological and ecological adaptive responses to acute or chronic hypoxic stress in humans who living at high attitude and who live at a normal sea level but suffer from hypoxic disorders.

\section{Conflict of Interest}

There is no conflict of interest.

\section{Acknowledgements}

This study was supported by Nature Science Foundation of Shaanxi Province, China (NO. 2016JM3024) and Nature Science Foundation of China (NO.31550008).

\section{References}

CERADINI DJ, KULKARNI AR, CALLAGHAN MJ, TEPPER OM, BASTIDAS N, KLEINMAN ME, CAPLA JM, GALIANO RD, LEVINE JP, GURTNER GC: Progenitor cell trafficking is regulated by hypoxic gradients through HIF-1 induction of SDF-1. Nat Med 10: 858-864, 2004.

CHEN X-Q, WANG S-J, DU J-Z, CHEN X-C: Diversities in hepatic HIF-1, IGF-I/IGFBP-1, LDH/ICD, and their mRNA expressions induced by $\mathrm{CoCl}_{2}$ in Qinghai-Tibetan plateau mammals and sea level mice. Am J Physiol Regul Integr Comp Physiol 292: R516-R526, 2007.

DU JZ, LI QF: Effects of simulated hypoxic acclimation on organism, organ and hematology in Ochotona curzoniae and rats. Acta Theriologica Sinica 2: 472-477, 1982.

GRANGE RW, MEESON A, CHIN E, LAU KS, STULL JT, SHELTON JM, WILLIAMS RS, GARRY DJ: Functional and molecular adaptations in skeletal muscle of myoglobin-mutant mice. Am J Physiol Cell Physiol 281: C1487-C1494, 2001.

GU Q, KONG Y, YU ZB, BAI L, XIAO YB: Hypoxia-induced SOCS3 is limiting STAT3 phosphorylation and NF-kappaB activation in congenital heart disease. Biochimie 93: 909-920, 2011. 
HOLMES K, ROBERTS OL, THOMAS AM, CROSS MJ: Vascular endothelial growth factor receptor-2: structure, function, intracellular signalling and therapeutic inhibition. Cell Signal 19: 2003-2012, 2007.

KIM Y-W, BYZOVA TV: Oxidative stress in angiogenesis and vascular disease. Blood 123: 625-631, 2014.

LIEBER RL: Skeletal Muscle Structure, Function, and Plasticity. Wolters Kluwer Health, Lippincott Williams\&Wilkins (eds), Baltimore, Maryland, USA, 2002, pp 116-200.

LIN J, WU H, TARR PT, ZHANG CY, WU Z, BOSS O, MICHAEL LF, PUIGSERVER P, ISOTANI E, OLSON EN: Transcriptional coactivator PGC-1a drives the formation of slow-twitch muscle fibres. Nature 418: 797-801, 2002.

MANALO DJ, ROWAN A, LAVOIE T, NATARAJAN L, KELLY BD, YE SQ, GARCIA JG, SEMENZA GL: Transcriptional regulation of vascular endothelial cell responses to hypoxia by HIF-1. Blood 105: 659-669, 2005.

PISANI DF, DECHESNE CA: Skeletal muscle HIF-1alpha expression is dependent on muscle fiber type. J Gen Physiol 126: $173-178,2005$.

SEMENZA GL: Hypoxia-inducible factor 1: oxygen homeostasis and disease pathophysiology. Trends Mol Med 7: 345-350, 2001.

STUTTFELD E, BALLMER-HOFER K: Structure and function of VEGF receptors. IUBMB Life 61: 915-922, 2009.

VOGT M, PUNTSCHART A, GEISER J, ZULEGER C, BILLETER R, HOPPELER H: Molecular adaptations in human skeletal muscle to endurance training under simulated hypoxic conditions. J Appl Physiol 91: 173-182, 2001.

XIE HC, HE JP, ZHU JF, LI JG: Expression of HIF-1alpha and VEGF in skeletal muscle of plateau animals in response to hypoxic stress. Physiol Res 63: 801-805, 2014. 